\title{
Effect of dietary fat and sucrose consumption on cardiac fibrosis in mice and rhesus monkeys
}

Niranjana Natarajan, ${ }^{1}$ Ana Vujic, ${ }^{1}$ Jishnu Das, ${ }^{2,3}$ Annie C. Wang, ${ }^{1}$ Krystal K. Phu, ${ }^{1}$ Spencer H. Kiehm, ${ }^{1}$ Elisabeth M. Ricci-Blair, ${ }^{1}$ Anthony Y. Zhu, ${ }^{1}$ Kelli L. Vaughan, ${ }^{4,5}$ Ricki J. Colman, ${ }^{6}$ Julie A. Mattison, ${ }^{4}$ and Richard T. Lee ${ }^{1,7}$

'Department of Stem Cell and Regenerative Biology, Harvard Stem Cell Institute, Harvard University, Cambridge, Massachusetts, USA. ${ }^{2}$ Ragon Institute of MCH, MIT and Harvard, Cambridge, Massachusetts, USA. ${ }^{3}$ Department of Biological Engineering, Massachusetts Institute of Technology, Cambridge, Massachusetts, USA. ${ }^{4}$ National Institute on Aging, NIH, Baltimore, Maryland, USA. ${ }^{5}$ SoBran BioSciences, SoBran Inc., Burtonsville, Maryland, USA. ${ }^{6}$ Department of Cell and Regenerative Biology and Wisconsin National Primate Research Center, University of Wisconsin-Madison, Madison, Wisconsin, USA. 'Cardiovascular Division, Department of Medicine, Brigham and Women's Hospital and Harvard Medical School, Boston, Massachusetts, USA.

Calorie restriction (CR) improved health span in 2 longitudinal studies in nonhuman primates (NHPs), yet only the University of Wisconsin (UW) study demonstrated an increase in survival in CR monkeys relative to controls; the National Institute on Aging (NIA) study did not. Here, analysis of left ventricle samples showed that CR did not reduce cardiac fibrosis relative to controls. However, there was a 5.9-fold increase of total fibrosis in UW hearts, compared with NIA hearts. Diet composition was a prominent difference between the studies; therefore, we used the NHP diets to characterize diet-associated molecular and functional changes in the hearts of mice. Consistent with the findings from the NHP samples, mice fed a UW or a modified NIA diet with increased sucrose and fat developed greater cardiac fibrosis compared with mice fed the NIA diet, and transcriptomics analysis revealed diet-induced activation of myocardial oxidative phosphorylation and cardiac muscle contraction pathways.

Authorship note: NN and AV are cofirst authors and contributed equally to this work.

Conflict of interest: RTL is a cofounder and member of the scientific advisory board of, and holds private equity in, Elevian, a company that aims to develop medicines to restore regenerative capacity. Elevian provides sponsored research support to the Lee Laboratory.

Copyright: (c) 2019, American Society for Clinical Investigation.

Submitted: March 7, 2019

Accepted: August 12, 2019

Published: September 19, 2019.

Reference information: JCI Insight.

2019;4(18):e128685.

https://doi.org/10.1172/jci.

insight.128685.

\section{Introduction}

Cardiovascular diseases are often age associated (1-4) and a leading cause of death worldwide (5). Cardiac fibrosis is a key feature of the aging heart and is linked to many forms of heart disease $(4,6,7)$. Increased deposition of extracellular matrix proteins (e.g., collagens) in the left ventricle (LV) leads to loss of compliance and diastolic dysfunction (8). There is increasing evidence to suggest that dietary composition may influence cardiac aging (9-12). With a deeper understanding of the relationship between diet composition and cardiac aging, we can better identify novel approaches to treat age-associated cardiac dysfunction.

Calorie restriction (CR), a chronic decrease in total daily caloric intake (13), delays the onset of most age-associated diseases and extends the mean and maximal life span of several model organisms, including yeast, worms, and rodents (14-18). The long-term effects of CR on cardiac health and survival are not fully understood. However, evidence from previous studies suggests that CR is associated with decreased incidence of atherosclerosis and oxidative stress in rodents $(19,20)$.

Rhesus macaques (Macaca mulatta) are the most commonly used nonhuman primate (NHP) species in biomedical aging research because they provide an excellent model for the study of human aging due to their genetic and physiological similarities. Furthermore, rhesus monkeys experience age-related physiological changes and disease progression similar to humans (21). The University of Wisconsin-Madison (UW) and the National Institute on Aging (NIA) have monitored the effects of CR on the health and survival of NHPs in separate longitudinal studies, both spanning more than 3 decades. The UW study reported a significant increase in survival of its calorie-restricted cohort, relative to its controls $(17,22)$, while the NIA study did not find any significant differences in the life span between its control and calorie-restricted cohorts (23). Table 1 summarizes the median life span of NHPs in both studies, and Table 2 shows the distribution of age at death for animals from which we analyzed samples in our study. 
Table 1. Survival median of NIA and UW NHP cohorts

\begin{tabular}{|c|c|c|c|}
\hline Site & Sex & Experimental group & $\begin{array}{l}\text { Survival median (yr) } \\
\text { (Kaplan-Meier) }\end{array}$ \\
\hline \multirow{2}{*}{ NIA } & \multirow{2}{*}{ Male, juvenile/adult } & Control & 28.78 \\
\hline & & CR & 26.31 \\
\hline & \multirow{2}{*}{ Male, old } & Control & 34.78 \\
\hline & & CR & 37.10 \\
\hline & \multirow{2}{*}{ Female, juvenile/adult } & Control & 25.67 \\
\hline & & CR & 22.63 \\
\hline & \multirow{2}{*}{ Female, old } & Control & 27.19 \\
\hline & & CR & 27.87 \\
\hline \multirow{4}{*}{ UW } & \multirow{2}{*}{ Male } & Control & 26.11 \\
\hline & & CR & 28.32 \\
\hline & \multirow{2}{*}{ Female } & Control & 23.86 \\
\hline & & CR & 29.68 \\
\hline
\end{tabular}

Adapted from Mattison et al. (21).

A comprehensive comparison of these studies noted methodological differences that may have contributed to the divergent effect on survival outcomes (24). There were 4 key differences between the NIA and UW studies: (a) diet composition, (b) genetic background, (c) feeding regimen, and (d) age at recruitment to the study (21). First, while the NIA study used a natural-ingredient diet, a fully defined, semipurified diet was used in the UW study. The UW diet had greater sucrose and fat content and lower fiber compared with the NIA diet (dietary composition of NIA and UW diets is summarized in Table 3). Second, the UW NHPs were of Indian origin and reared on-site, whereas the NIA study used a mixture of Indian- and Chinese-origin NHPs obtained from various sources. Third, the NIA study administered 2 meals per day, 1 at 6:30 am and another at $1 \mathrm{pm}$ along with a low-calorie treat (fruit). The UW study administered 1 meal at 8 am and a fruit/ vegetable treat at $4 \mathrm{pm}$ while removing any remaining food. Finally, the NIA study recruited NHPs in 3 age groups: juvenile (1-2 years), adolescent/young adult (3-5 years for males and 6-14 years for females), and old (16-23 years). The UW study recruited adult animals to its study, approximately 7-14 years of age. Animal housing conditions, temperature, light cycles, water, and routine animal monitoring by veterinary staff were equivalent at both facilities (21). Although these studies had important design differences, taken together, they reveal that the health benefits of CR are conserved in NHPs, and thus, may be translatable to humans (21).

Age-associated cardiac changes in NHPs approximate those of humans, and as such, samples from the 2 long-term NHP studies present a unique opportunity to further explore cardiac aging and factors that may explain some of the different outcomes between the 2 NHP studies. To this end, LV tissue samples from both the NIA and UW studies were analyzed to investigate cardiac fibrosis. Further, we extended findings from the NHP studies and conducted a rodent experiment using the NHP diets to characterize cardiac fibrosis and the molecular and functional changes in the heart that are associated with alterations in diet. We hypothesized that dietary composition may be an important factor associated with cardiac fibrosis and aimed to better characterize this relationship.

\section{Results}

Long-term CR, diet composition, and cardiac fibrosis in NHPs. Long-term CR in rodent models has been shown to decrease cardiac fibrosis induced by high-fat and "Western" diets (25). To test this hypothesis in NHPs, fibrosis was quantified in both NIA and UW LV tissue samples using Masson's trichrome staining and subsequent blinded image analyses of 3 discrete sections from each LV (26) (representative images are shown in Figure 1A). Total cardiac fibrosis was measured as previously described (27), and, to avoid image selection bias, entire sections were imaged for subsequent analysis. A representative image of whole stained LV sections from an NIA and UW NHP is shown in Supplemental Figure 1 (supplemental material available online with this article; https://doi.org/10.1172/jci. insight.128685DS1). Range of age at death for the samples analyzed is summarized in Table 2. Upon comparing age at death, there was no significant difference between the groups (Supplemental Table 1). 
Table 2. Age distribution of NHP heart samples analyzed in this study

\begin{tabular}{|c|c|c|c|c|}
\hline Site & Sex & Control (n) & $\operatorname{CR}(n)$ & Age at death (range, in yr) \\
\hline \multirow{2}{*}{ NIA } & Male & 15 & 11 & $\begin{array}{c}25.7-41.2 \text { (Control) } \\
21.5-44.2(\mathrm{CR})\end{array}$ \\
\hline & Female & 9 & 7 & $\begin{array}{c}20.7-33.8 \text { (Control) } \\
17.8-36.0(\mathrm{CR})\end{array}$ \\
\hline UW & Male & 17 & 15 & $\begin{array}{c}21.3-30.5 \text { (Control) } \\
21.3-35.1 \text { (CR) }\end{array}$ \\
\hline
\end{tabular}

Here, CR did not significantly alter the percentage of myocardial area occupied by fibrosis in either NIA or UW NHPs (Figure 1B), but there was a striking difference in myocardial fibrosis between the NIA and UW NHPs. In a comparison by diet group, NIA controls had significantly less fibrosis relative to the UW controls. Similarly, the NIA CR NHPs had significantly less fibrosis compared with UW CR NHPs (Figure 1B, overall $P<0.001$ using a Kruskal-Wallis test; $P<0.001$ for NIA control vs. UW control, NIA control vs. UW CR, NIA CR vs. UW CR, and NIA CR vs. UW control using post hoc pairwise 2-sided Mann-Whitney $U$ tests adjusted for multiple comparisons using a Bonferroni's correction). There was no effect of CR when the groups were evaluated by sex (Figure 1C, overall $P<0.001$ using a Kruskal-Wallis test; $P<0.001$ for NIA control male vs. UW control male, NIA control female vs. UW control female, NIA CR male vs. UW CR male, and NIA CR female vs. UW CR female using post hoc pairwise 2-sided Mann-Whitney $U$ tests adjusted for multiple comparisons using a Bonferroni's correction). Yet, again, we found that UW male and female NHPs had significantly greater cardiac fibrosis compared with NIA counterparts (Figure 1C). Finally, in a comparison of percentage of cardiac fibrosis between site cohorts, UW LV samples had a 5.9-fold higher median percentage of fibrosis compared with NIA samples (Figure 1D, $P<0.001$ using a 2-sided Mann-Whitney $U$ test). The pattern of fibrosis in the NHP LV tissues from NIA and UW was primarily interstitial fibrosis, as seen in Figure 1A.

The integrity of the extracellular matrix (ECM) and its structure is maintained by a balance in the function of matrix metalloproteinases (MMPs) and tissue inhibitor of metalloproteinases (TIMPs) (28). Classically, MMPs degrade ECM components, mediating tissue remodeling and maintenance, while TIMPs inhibit MMP activity, restricting ECM degradation and thereby promoting ECM deposition (28, 29). To better understand ECM dynamics in NHP hearts from the NIA and UW studies, we assessed the

Table 3. Dietary composition of the 4 study diets: chow, NIA, UW, and mNIA

\begin{tabular}{|c|c|c|c|c|c|c|c|c|c|c|c|c|}
\hline \multirow[b]{2}{*}{ Ingredients } & \multicolumn{3}{|c|}{$\begin{array}{c}\text { Chow } \\
\text { (caloric density } 3.46 \mathrm{kCal} / \mathrm{g} \text { ) }\end{array}$} & \multicolumn{3}{|c|}{$\begin{array}{c}\text { NIA diet } \\
\text { (caloric density } 3.418 \mathrm{kCal} / \mathrm{g} \text { ) }\end{array}$} & \multicolumn{3}{|c|}{$\begin{array}{c}\text { UW diet } \\
\text { (caloric density } 3.927 \text { kCal/g) }\end{array}$} & \multicolumn{3}{|c|}{$\begin{array}{c}\text { mNIA diet } \\
\text { (caloric density } 3.584 \mathrm{kCal} / \mathrm{g} \text { ) }\end{array}$} \\
\hline & $\begin{array}{l}\% \text { by } \\
\text { weight }\end{array}$ & $\% \mathrm{kCal}$ & Sources & $\begin{array}{l}\% \text { by } \\
\text { weight }\end{array}$ & $\% \mathrm{kCal}$ & Sources & $\begin{array}{l}\% \text { by } \\
\text { weight }\end{array}$ & \% kCal & Sources & $\begin{array}{l}\% \text { by } \\
\text { weight }\end{array}$ & $\% \mathrm{kCal}$ & Sources \\
\hline Protein & 22.5 & 26.3 & $\begin{array}{l}\text { Soybean, } \\
\text { fish meal }\end{array}$ & 17.5 & 19.7 & $\begin{array}{l}\text { Soybean, } \\
\text { fish meal }\end{array}$ & 13.1 & 13.4 & Lactalbumin & 13.5 & 15.06 & $\begin{array}{l}\text { Soybean, } \\
\text { fish meal }\end{array}$ \\
\hline Sucrose & 1.03 & 0.8 & & 3.9 & 7.6 & & 28.5 & 46 & & 26.81 & 29.9 & \\
\hline Fat & $5.5-6.8$ & 14.3 & $\begin{array}{l}\text { Soy, corn, } \\
\text { porcine } \\
\text { animal } \\
\text { fat }\end{array}$ & 7 & 17.7 & $\begin{array}{l}\text { Soy, corn, } \\
\text { fish oils }\end{array}$ & 10.6 & 24.4 & Corn oil & 10.6 & 26.6 & $\begin{array}{l}\text { Soy, corn, } \\
\text { fish oils }\end{array}$ \\
\hline Fiber & 4 & & Cellulose & 6.7 & & Cellulose & 5 & & Cellulose & 8.3 & & Cellulose \\
\hline
\end{tabular}


A
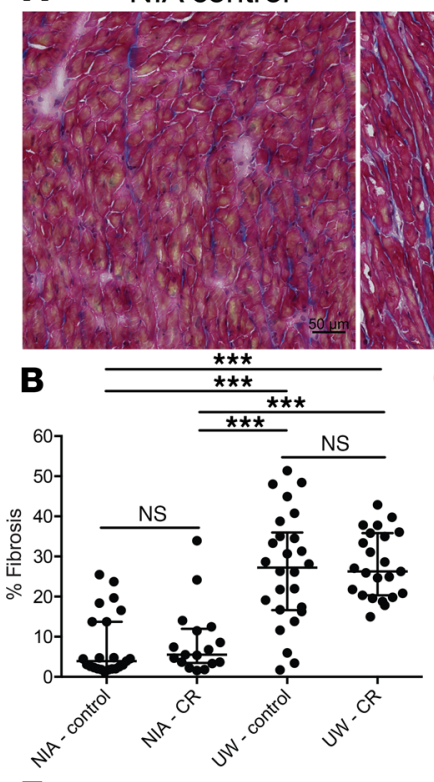

NIA CR
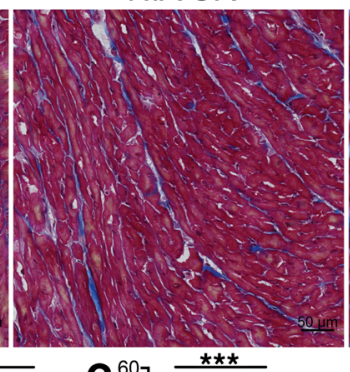
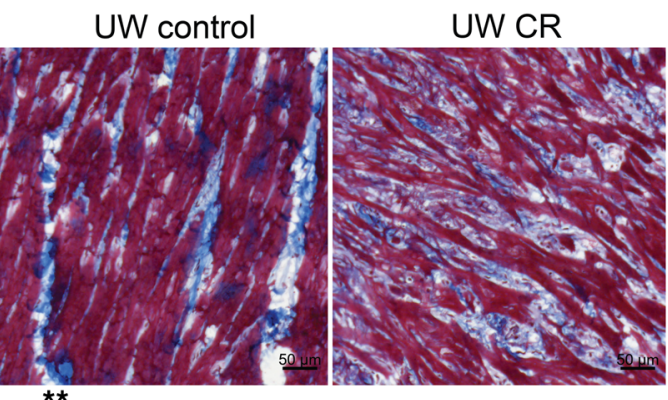

D

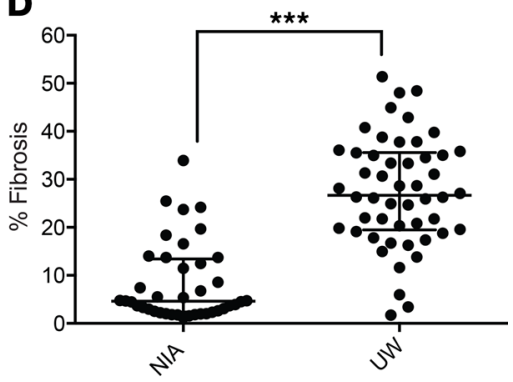



Figure 1. In NHPs, cardiac fibrosis is more abundant in UW animals compared with those from NIA. However, no significant difference in cardiac fibrosis was observed between control and CR samples in either cohort. (A) Representative Masson's trichrome-stained NHP LV tissues from control and CR groups of the NIA and UW studies show greater extent of cardiac fibrosis in the UW cohort. Scale bar: $50 \mu \mathrm{m}$. (B) Cardiac fibrosis was not significantly affected by CR in the NIA and UW cohorts. $P=$ n.s. (nonsignificant), and ${ }^{* *} P<0.001$ by Kruskal-Wallis test followed by pairwise Mann-Whitney $U$ tests with appropriate Bonferroni's corrections to adjust for multiple comparisons. In the figure panel, pairwise significance/lack of significance is indicated based on $P$ values from Mann-Whitney $U$ test. (C) Percentage of myocardial area occupied by fibrosis was evaluated by sex and dietary regimen. Fibrosis within each study was not significantly different between control and CR groups when matched by sex. There was greater fibrosis in UW control and CR, compared with NIA cohorts. ${ }^{* *} P<0.01$; ${ }^{* *} P<0.001$, by Kruskal-Wallis test followed by pairwise Mann-Whitney $U$ tests with appropriate Bonferroni's corrections to adjust for multiple comparisons. In the figure panel, pairwise significance/lack of significance is indicated based on $P$ values from Mann-Whitney $U$ test. (D) Total cardiac fibrosis was significantly greater in UW LVs compared with NIA. ${ }^{* * *} P<0.001$, Mann-Whitney $U$ test. Scatter plots to represent the data are shown with median with interquartile range. $n:$ NIA: total $n=42,(24$ control [15 male, 9 female]; 18 CR [11 male, 7 female]); UW: total $n=49$ (26 control [16 male, 10 female]; 23 CR [14 male, 9 female]). (E) Heatmap of normalized expression values of specific genes in NHP heart samples from NIA and UW studies as measured from NanoString experiments. Median normalized abundances of genes assayed are shown in rows, and the columns represent each of the 4 cohorts: NIA control, UW control, NIA CR, and UW CR. Significant differences and trends among the groups are indicated by yellow boxes around pairwise comparisons; ${ }^{*} P<0.05$ pairwise 2 -tailed $t$ test with Benjamini-Hochberg FDR correction. $n=6$ for all groups. 
expression of MMPs and TIMPs in NHP heart samples using nCounter gene expression analyses (30). Six control and $6 \mathrm{CR}$ samples each were analyzed from the NIA and UW cohorts. Interestingly, we found significantly greater expression of TIMP2 and TIMP4 in the NIA CR hearts, compared with the UW CR cohort (Figure 1E, $P<0.05$, 2-tailed $t$ test with Benjamini-Hochberg FDR correction). There were no significant differences in TIMP2 and TIMP4 between NIA control and UW control samples $(P=$ nonsignificant, 2-tailed $t$ test with Benjamini-Hochberg FDR correction). High levels of TIMP2 and TIMP4 have been documented in the heart, and their expression is dysregulated in various cardiovascular diseases (31-33). Furthermore, there was a trend in the levels of collagen type III (greater expression in UW CR, compared with NIA CR) and MMP2 (greater expression in NIA CR, compared with UW CR, Figure $1 \mathrm{E})$. There were no significant differences in the expression of the other genes assayed (Figure 1E, $P=$ nonsignificant, 2-tailed $t$ test with Benjamini-Hochberg FDR correction).

Long-term consumption of moderate-fat, high-sucrose diet is associated with cardiac fibrosis in male mice. One of the key study design differences between the NIA and UW NHP studies is the composition of diets administered (24). The UW study used a semipurified, defined diet with higher levels of sucrose and fat ( $28 \%$ sucrose, $10.6 \%$ fat by weight), while the NIA study provided a complex, natural-ingredient diet containing fish oils ( $3.9 \%$ sucrose, $5 \%$ fat by weight, Table 3 , dietary composition). To determine whether the increased sucrose and fat content in the UW diet contributed to the increase in cardiac fibrosis, 6-monthold male C57BL/ 6 mice were randomized into 1 of 4 diet groups: standard mouse chow $(n=10)$, the UW diet $(n=15)$, the NIA diet $(n=12)$, or the NIA diet modified (mNIA) $(n=8)$ to include increased levels of sucrose and fat equivalent to the UW diet for 6 months (Supplemental Figure 2).

After 6 months, NIA-fed mice weighed significantly less than all 3 other groups, chow, UW, and mNIA (Figure 2A, overall $P<0.01$ using a Kruskal-Wallis test; $P<0.01$ for body weights on chow vs. NIA, UW vs. NIA, and mNIA vs. NIA using post hoc pairwise 2-sided Mann-Whitney $U$ tests adjusted for multiple comparisons using a Bonferroni's correction). Moreover, 5-hour-fasted blood glucose levels were significantly higher in UW and mNIA cohorts compared with levels of those on chow and NIA (Supplemental Figure 2B, overall $P<0.01$ using a Kruskal-Wallis test; $P<0.05$ for fasting glucose on chow vs. UW, NIA vs. UW, and mNIA vs. NIA using post hoc pairwise 2-sided Mann-Whitney $U$ tests adjusted for multiple comparisons using a Bonferroni's correction). Five-hour fasting plasma insulin was significantly greater in the mNIA cohort, compared with the NIA cohort (Supplemental Figure 2C, overall $P<0.05$, Kruskal-Wallis test; $P<$ 0.05 , NIA vs. mNIA, post hoc Mann-Whitney $U$ test with Bonferroni's multiple-testing correction). We estimated hepatic and plasma triglycerides in our cohorts. Hepatic triglyceride content was significantly greater in the mNIA cohort compared with the other 3 diet cohorts and in the UW cohort was greater than the NIA cohort (Supplemental Figure 2D, overall $P<0.0001,1$-way ANOVA; $P<0.05$ for NIA vs. UW and UW vs. mNIA; $P<0.01$ for chow vs. mNIA; $P<0.001$ for NIA vs. mNIA; post hoc 2 -tailed $t$ test with Bonferroni's multiple-testing correction). However, there were no significant differences in plasma triglyceride content between the dietary cohorts (Supplemental Figure 2E). These data indicate that long-term consumption of high-sucrose diets can lead to insulin resistance and greater lipid accumulation in the liver. In addition, heart weight to body weight ratios were significantly lower in the mNIA cohort compared with chow and NIA cohorts (Figure 2B, overall $P<0.05$ using a Kruskal-Wallis test; $P<0.05$ for heart weight to body weight ratios on chow vs. mNIA and mNIA vs. NIA using post hoc pairwise 2-sided Mann-Whitney $U$ tests adjusted for multiple comparisons using a Bonferroni's correction). Lower heart weight to body weight ratios in the mNIA cohort were consistent with a recent study in rats fed a high-fat diet for 24 weeks (34), and they point toward inadequate compensation of heart weight to body weight in the context of obesity $(25,35)$. Representative images of Masson's trichrome-stained samples are shown in Figure 2C. mNIA and UW mice had significantly greater total cardiac fibrosis than chow and NIA (Figure 2D, overall $P<0.05$ using a Kruskal-Wallis test; $P<0.05$ for percentage of fibrosis on chow vs. NIA; $P<0.01$ for percentage of fibrosis on chow vs. mNIA; $P<0.001$ for percentage of fibrosis on chow vs. UW, NIA vs. UW, and NIA vs. mNIA using post hoc pairwise 2-sided Mann-Whitney $U$ tests adjusted for multiple comparisons using a Bonferroni's correction). The pattern of fibrosis observed in the mouse hearts was primarily perivascular, and it was most prominent with the UW and mNIA-fed groups. This pattern was different in the NHP hearts and was primarily interstitial fibrosis (7), as seen in Figure 1A (NHP) and Figure 2C (mice). This increase in fibrosis in a rodent model resulting from long-term consumption of high sucrose and fat corroborates the finding of increased cardiac fibrosis in the UW NHP cohort (Figure 1D). Thus, these data support our hypothesis that dietary composition likely plays a key role in cardiac fibrosis, and this effect is conserved in mice and NHPs. 

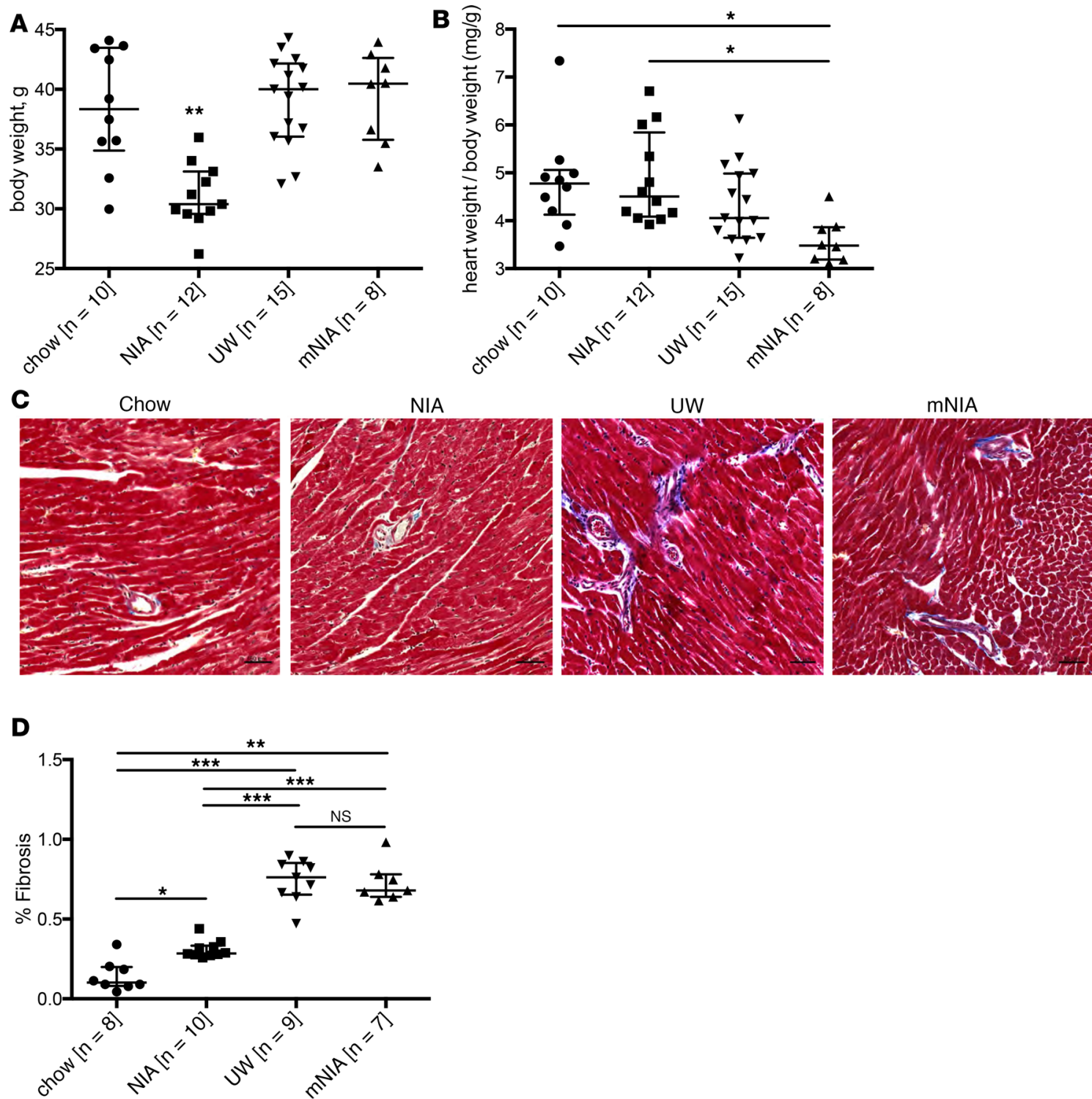

Figure 2. Mice fed the UW or the mNIA diet for 6 months have elevated body weights, higher fasting glucose levels, and increased cardiac fibrosis. (A) Body weights of mice fed NIA diet for 6 months were significantly lower than chow, UW, and mNIA diet groups. ${ }^{* *} P<0.01$, by Kruskal-Wallis test followed by pairwise Mann-Whitney $U$ tests with appropriate Bonferroni's corrections to adjust for multiple comparisons. In the figure panel, pairwise significance/ lack of significance is indicated based on $P$ values from Mann-Whitney $U$ test. Scatter plots to represent the data are shown with median with interquartile range. (B) Heart weight to body weight ratios of mice in the 4 diet cohorts. ${ }^{*} P<0.05$, by Kruskal-Wallis test followed by pairwise Mann-Whitney $U$ tests with appropriate Bonferroni's corrections to adjust for multiple comparisons. In the figure panel, pairwise significance/lack of significance is indicated based on $P$ values from Mann-Whitney test. Scatter plots to represent the data are shown with median with interquartile range. (C) Representative Masson's trichrome images of hearts from chow, NIA, UW, and mNIA diet groups (scale bar: $50 \mu \mathrm{m}$ ). Increased cardiac fibrosis observed in UW diet- and mNIA diet-fed mice was predominantly perivascular. (D) Percentage of fibrosis of UW and mNIA diet cohorts was significantly greater compared with chow and NIA diet cohorts. Overall $P<0.001$, by Kruskal-Wallis test. In the figure panel, pairwise significance/lack of significance is indicated based on $P$ values from Mann-Whitney $U$ tests with appropriate Bonferroni's corrections to adjust for multiple comparisons. $P=$ n.s. (nonsignificant); ${ }^{*} P<0.05 ;{ }^{* *} P<0.01 ;{ }^{* *} P<0.001$. Scatter plots to represent the data are shown with median with interquartile range.

Systematic characterization of diet-induced alterations to global transcriptional profiles. Molecular changes in the LV mouse tissue were assessed using a transcriptomic approach. Total poly-adenylated mRNA was isolated and sequenced on an Illumina NextSeq flow cell in high-output mode to generate approximately 300 million $1 \times 75$ bp reads. The reads were aligned to the mouse transcriptome (36), and gene expression levels were quantified using an expectation-maximization approach (37). Using this pipeline, we obtained global transcriptome-wide gene expression levels for each of the diets.

Next, we examined overall transcriptomic profiles for the 3 primary diets: chow, UW, and NIA. Using t-distributed stochastic neighbor embedding (t-SNE) (38), we visualized the entire high-dimensional 
transcriptomic profiles in 2 dimensions that best summarized the overall profiles. Because t-SNE is an unsupervised approach, the dimensionality reduction did not account for diet group. Thus, it provided an unbiased summary to contrast global transcriptional profiles from mice on the study diets, which varied in sucrose and fat content. These results indicated that in terms of global transcriptomic profiles, the UW and NIA diets induced distinct signatures (Figure 3A), while chow and NIA diets had a similar transcriptional profile. This result was robust across t-SNE technical replicates (Figure 3A and Supplemental Figure 3A). We also obtained the same trend with a principal component analysis (PCA) (Supplemental Figure 3B). These findings are consistent with the expectation that a semipurified diet (UW) and a natural-ingredient diet (NIA) induce distinct transcriptomic profiles. Further, because chow and NIA are both natural-ingredient diets, it is unsurprising that mice on these diets have similar transcriptomic signatures.

Though t-SNE provides an excellent summary of distinct clusters, relative distances do not have a Cartesian interpretation in a high-dimensional embedding. Conversely, relative distances in a PCA do have an intuitive interpretation, permitting exploration into relative relationships of the mNIA diet to the 3 primary diets. PCA-based visualization was consistent with our previous findings (Figure 3B); we observed that the transcriptional profiles induced by the chow and the NIA diets were distinct from the profile induced by the UW diet. Interestingly, the mNIA diet induced a global signature that had some similarities to the UW profile, as well as to the chow/NIA profile. This implies that merely examining the global mNIA profile is insufficient in explaining the complex and specific transcriptional changes occurring with the addition of fat and sucrose to a natural-ingredient diet.

Thus, to thoroughly characterize the nature of these diet-induced alterations, we first focused on changes at the level of individual transcripts across the diets. Using DESeq2 (39), we identified transcripts that were significantly differentially expressed (both in terms of an effect size cutoff and a statistical significance threshold; see Methods for details) between each pair of diets (Figure 3C). Our results were robust to the choice of alignment algorithm (Supplemental Figure 3B) and whether gene- or transcript-level quantifications were used (Supplemental Figure 3C). Although there were differences across all diets, the most dramatic differences were between the mNIA and chow diets and between mNIA and NIA diets (Figure 3D). These data indicate that there were effectively 3 distinct profiles associated with characteristics of the 4 different diets: (a) semipurified, defined diets (UW); (b) natural-ingredient diets (chow and NIA); and (c) the addition of fat and sucrose to an otherwise-healthy natural-ingredient diet (mNIA) (Table 3, dietary composition).

Diet-induced alterations at the functional level. Functional changes induced by variations in diet were identified by entire gene sets/processes that were most significantly differentially transcriptionally regulated across the diets. Specifically, we examined overrepresentation (40) of the most significantly differentially expressed genes, as defined based on both a fold change cutoff and a stringent FDR threshold, in Kyoto Encyclopedia of Genes and Genomes (KEGG) pathways (41), i.e., a set of biological pathways defined based on prior knowledge. We used overrepresentation analyses on gene sets identified using stringent cutoffs to focus on the most dramatic transcriptional changes diet induced. These analyses allow the systematic and unbiased identification of pathways corresponding to those in which the greatest transcriptomic changes occur, after looking across all KEGG pathways.

Genes with the highest expression levels from mNIA-fed mice, as compared with NIA and chow diet groups, were significantly overrepresented in pathways involved in cardiac muscle contraction and catabolic processes, including oxidative phosphorylation and metabolism (Figure 4 and Supplemental Table 2). Conversely, genes with higher expression levels in mice on the NIA diet, compared with the mNIA diet, were significantly enriched in more anabolic processes, such as fatty acid biosynthesis. Thus, when fat and sucrose were added, even to a natural-ingredient diet, gene expression related to cardiac contraction and metabolism was dramatically altered.

Next, to obtain an unbiased assessment of any functional changes associated with diet, we characterized protein-protein interaction network modules that differed across diets. Using a database of well-characterized Mus musculus protein interactions (42) (defined as those supported by 2 or more independent experimental assays), we identified network modules centered on proteins encoded by genes that were differentially expressed among chow, mNIA, and NIA diets. Protein network modules have often been used to move beyond examining individual interactions to a more holistic understanding of how coordinated functional changes occur (43-46). Here, protein network modules involving genes with higher expression in hearts of animals on an mNIA diet, as compared with chow and NIA, primarily included (a) signaling 


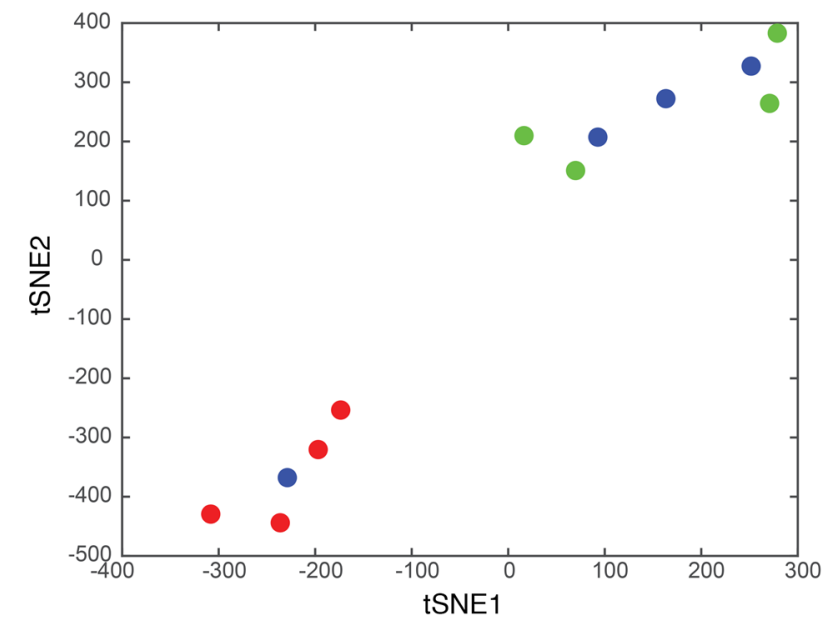

A modified-NIA diet $\mathbf{B}$

\section{C}
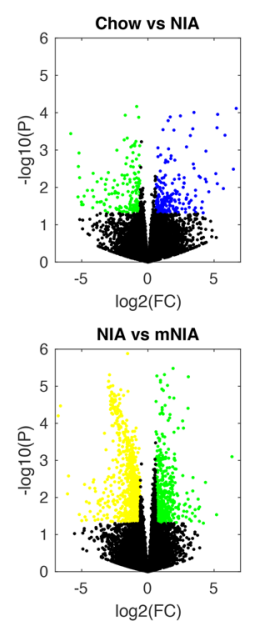
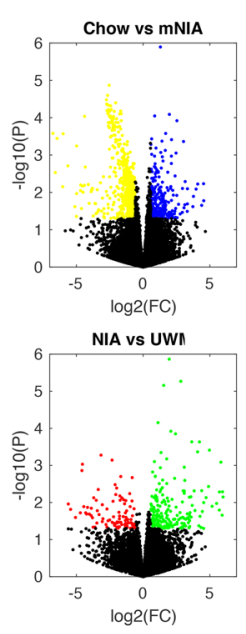
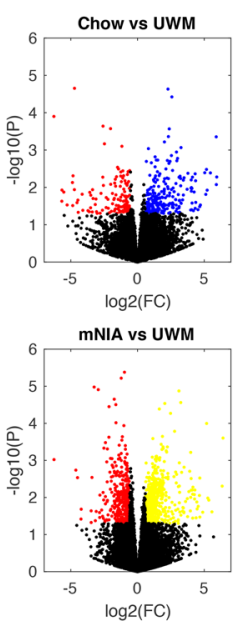

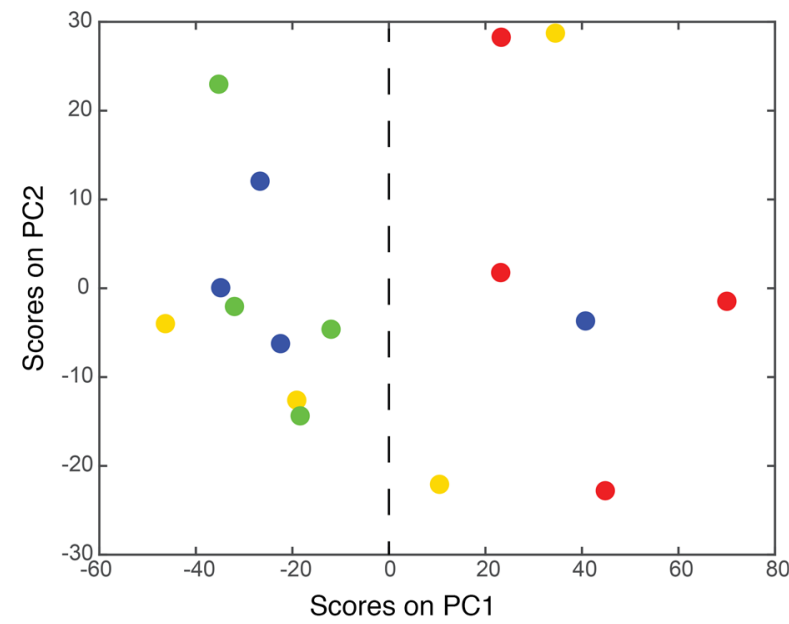

D

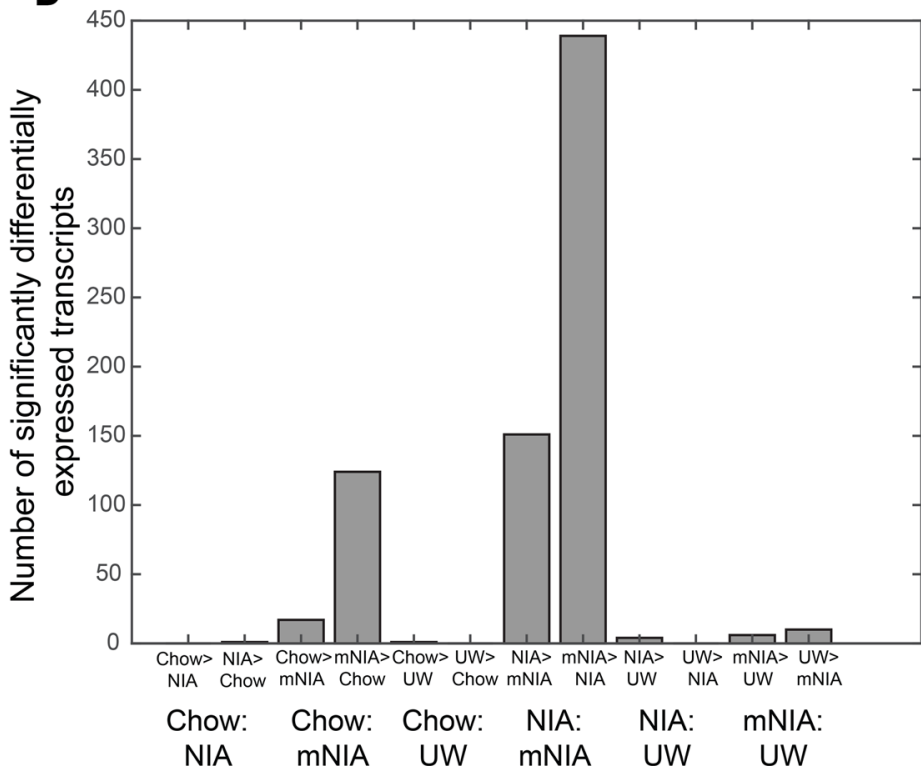

Figure 3. In mice, diet composition is associated with distinct transcriptional profiles. (A) t-SNE visualization of global transcriptomic profiles (i.e., all expression levels of all transcripts) of hearts from mice fed chow, NIA, or UW diets. (B) PCA visualization of global transcriptomic profiles (i.e., all expression levels of all transcripts) of hearts from mice fed chow, NIA, UW, or mNIA diets. (C) Volcano plots illustrating transcripts that are differentially expressed between each pair of diets. (D) Bar graphs illustrating numbers of significantly differentially expressed transcripts between each pair of diets.

cascades, (b) transcriptional regulators, and (c) innate cardioprotective mechanisms that limit remodeling (Figure 5, A and B). These findings further support the notion that the mNIA diet induced cardiac stress, but it may have also triggered innate cardioprotective mechanisms to counter that stress.

Interestingly, protein network modules involving genes significantly higher in mNIA compared with chow (Figure 5A) resembled modules involving genes significantly higher in mNIA compared with NIA (Figure $5 \mathrm{~B}$ ). This suggests that the mNIA diet was "similarly different" from both the NIA and the standard chow diet and that the addition of fat and sucrose had negative consequences relative to both diets. This is also consistent with our previous findings that the chow and NIA diets themselves induced similar transcriptomic profiles (Figure 3, A and B); hence the mNIA diet induced a profile that was "similarly different" from both. On the other hand, modules involving genes significantly higher in NIA compared with mNIA were primarily associated with cardiovascular homeostasis (Figure 5C). Thus, these analyses present an unbiased perspective of how changes in diet have important functional consequences. Further, of these 3 diets, the mNIA 


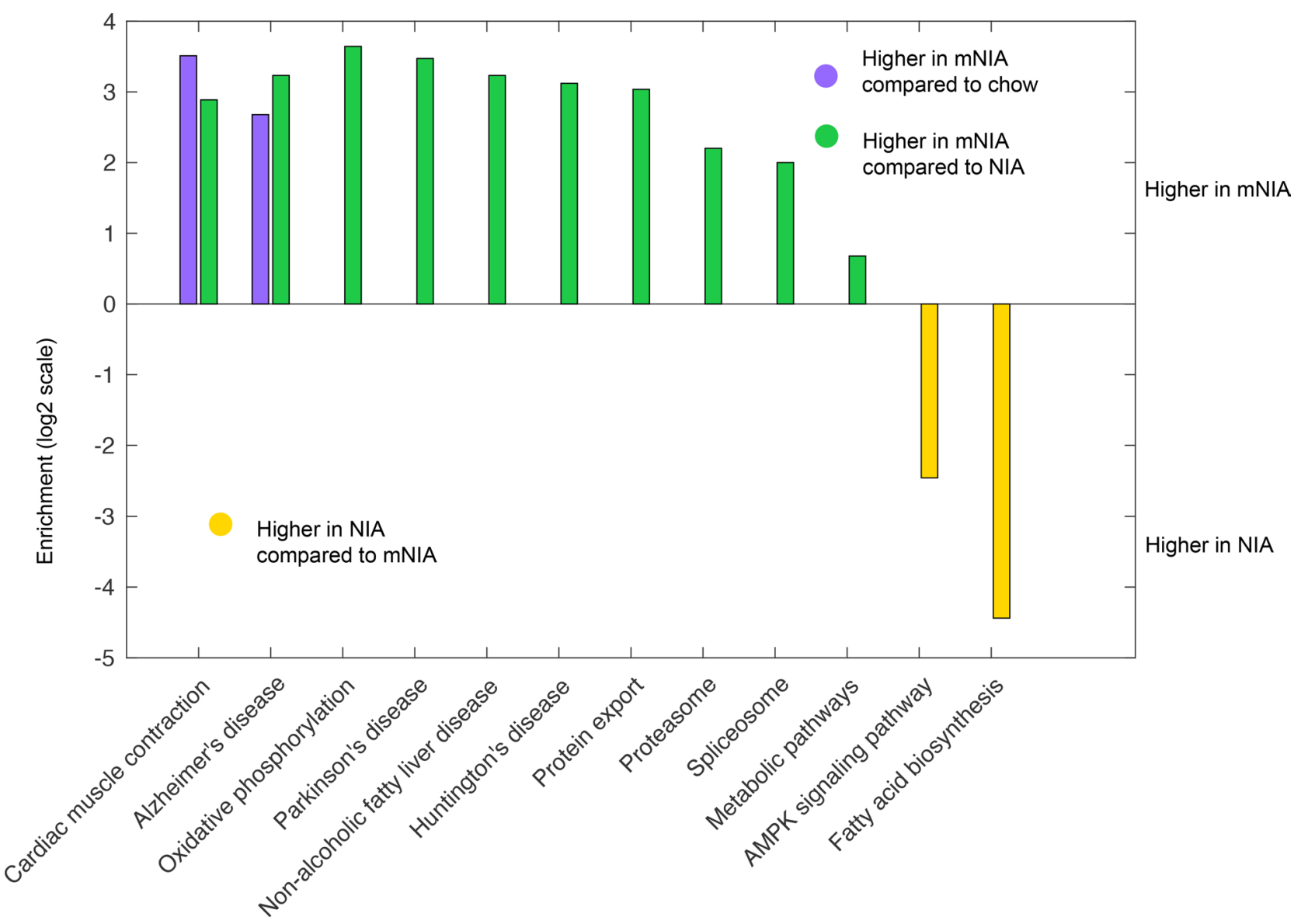

Figure 4. Summary of KEGG pathways in which significantly differentially expressed genes (mNIA vs. chow, mNIA vs. NIA, and NIA vs. mNIA) are overrepresented.

diet resulted in the greatest percentage of fibrosis (Figure 2D). Transcriptomic analyses showed that rodents consuming the mNIA diet triggered cardioprotective mechanisms in response to cardiac and oxidative stress. Thus, both the histological and transcriptomic data consistently suggest that among the different diets in this study, the mNIA diet was the most deleterious for normal cardiac function.

Finally, we explored whether there were any conserved upstream regulatory mechanisms that drove these changes in transcriptional profiles. Specifically, we used a high-quality genome-scale mouse transcriptional regulatory network (curated systematically from small-scale as well as high-throughput experiments) (47) to identify transcription factors upstream of genes that had significantly higher expression in the mNIA diet compared with the chow and NIA diets. There were 38 and 71 transcription factors (TFs) upstream of genes with significantly higher expression in the mNIA diet compared with the chow and NIA diets, respectively. We performed overrepresentation analyses and found these TFs significantly overrepresented (FDR < 0.05) in several KEGG pathways. Interestingly, the 38 TFs upstream of genes with significantly higher expression in the mNIA diet compared with the chow diet were significantly overrepresented in the Th17 cell differentiation pathway $(5$ genes, FDR $=0.003)$ and in the advanced glycation end products/ receptor for advanced glycation end products (AGE/RAGE) signaling pathway (5 genes, FDR $=0.0003$ ). AGEs are generated because of chronic hyperglycemia and promote cardiovascular disease progression via direct RAGE-dependent mechanisms $(48,49)$. AGEs accumulate in the heart and coronary blood vessels to induce oxidative stress, inflammation, and ECM accumulation, leading to increased cardiac fibrosis and compromise in function $(48,49)$. Therefore, we postulate that AGE/RAGE signaling could explain the increase in fibrosis observed on the mNIA diet. The 71 TFs upstream of genes with significantly higher expression in the mNIA diet compared with the NIA diet were also significantly overrepresented in the Th17 cell differentiation pathway ( 5 genes, FDR $=0.0005)$. Th17/Treg imbalances have been reported in patients with heart failure - both heart failure with preserved ejection fraction ( $\mathrm{HFpEF})$ and heart failure 
JClinsIGHT

RESEARCH ARTICLE

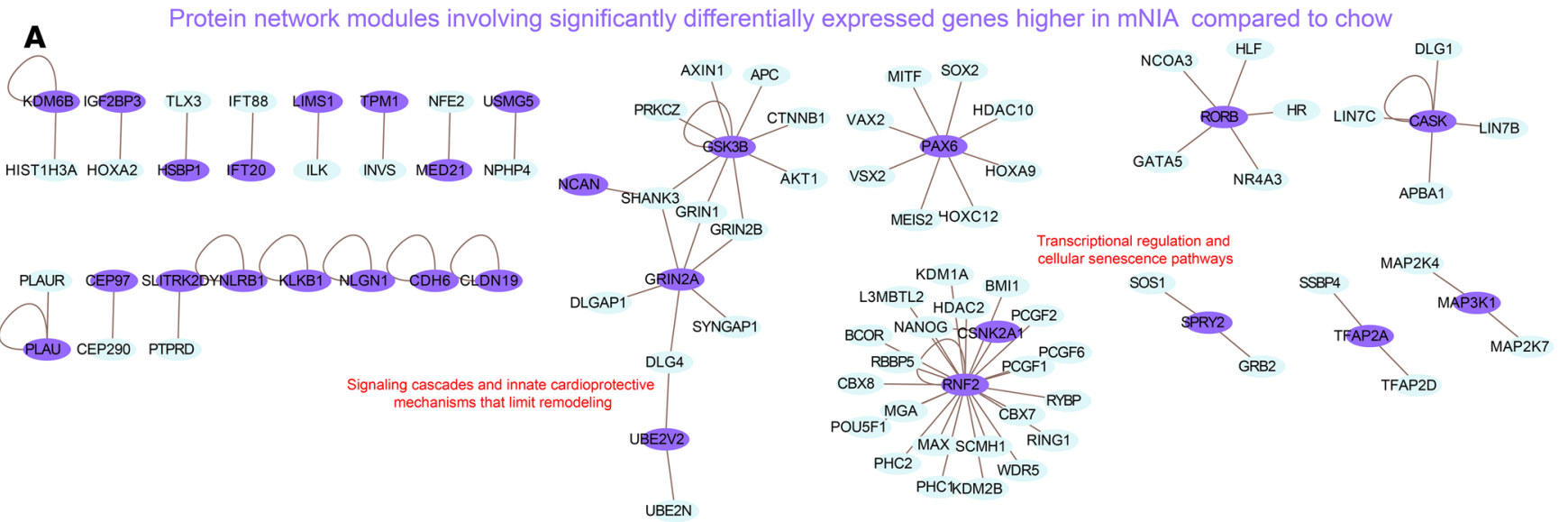

B Protein network modules involving significantly differentially expressed genes higher in mIA compared to NIA

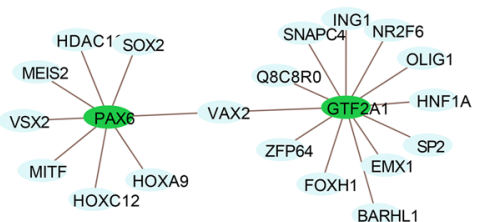

Transcriptional regulation pathways
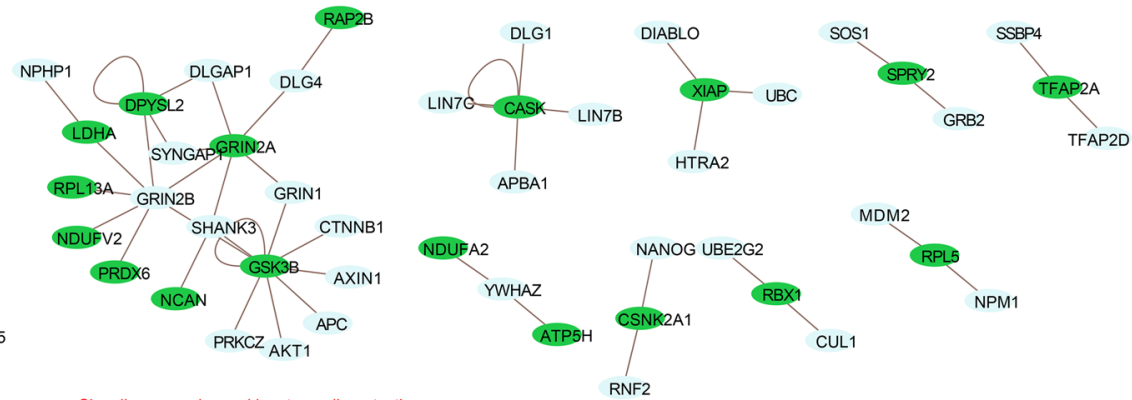
Signaling cascades and innate cardioprotectiv
mechanisms that limit remodeling
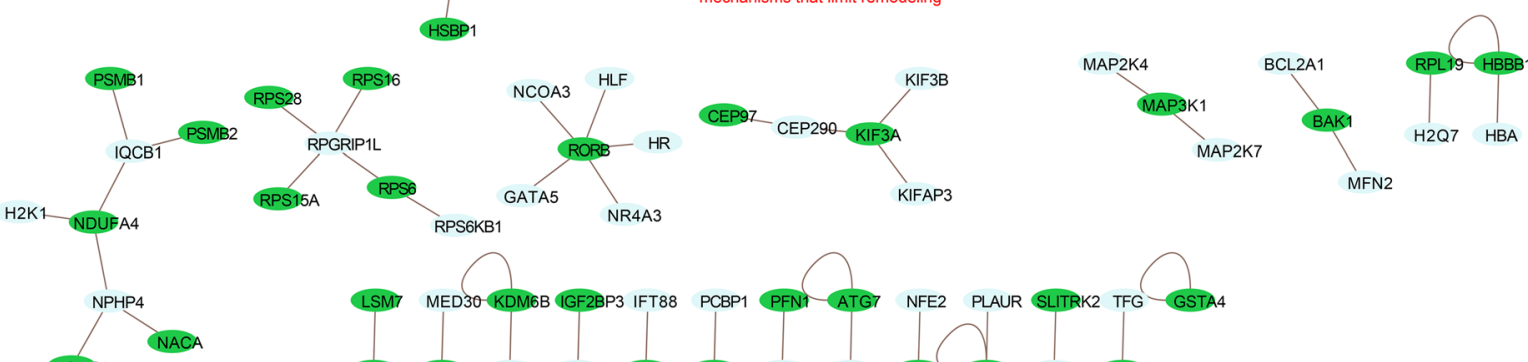

C

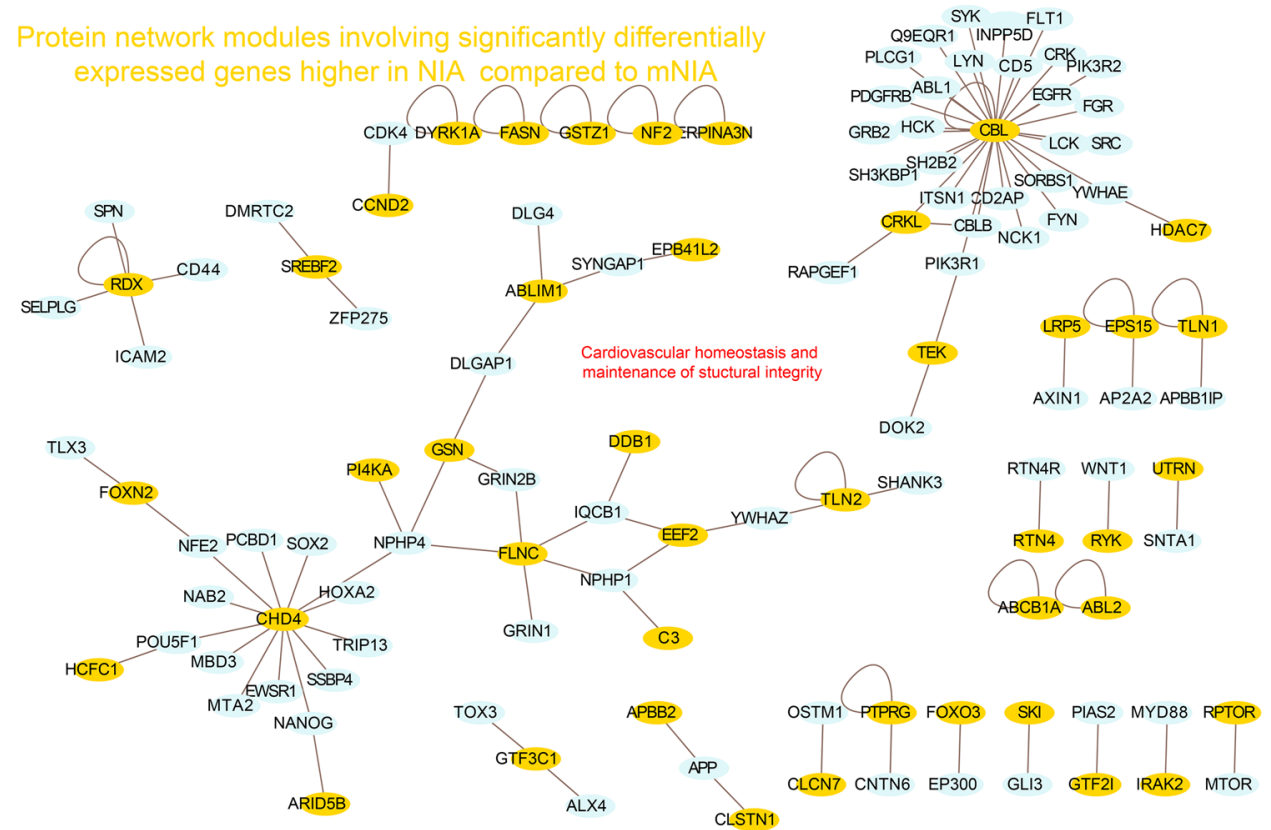

insight.jci.org https://doi.org/10.1172/jci.insight.128685

10 
Figure 5. Pathway and protein network module analyses to identify key functional signatures that are upregulated in mNIA, compared with chow and NIA. (A) Protein network modules involving significantly differentially expressed genes that have higher expression levels in mNIA compared with chow. (B) Protein network modules involving significantly differentially expressed genes that have higher expression levels in mNIA compared with NIA. (C) Protein network modules involving significantly differentially expressed genes that have higher expression levels in NIA compared with mNIA.

with reduced ejection fraction (50). Therefore, inflammation and the Th17 cell pathway could also be playing a role in the progression of cardiovascular disease due to chronic consumption of high dietary sucrose.

Next, because our lists of differentially expressed genes themselves included some TFs, we explored downstream targets of these TFs. We found 78 and 67 genes downstream of TFs with significantly higher expression in the mNIA diet compared with the chow and NIA diets, respectively. These genes were also significantly overrepresented (FDR < 0.05) in a number of KEGG pathways (Supplemental Table 3). Intriguingly, the 78 genes downstream of TFs with significantly higher expression in the mNIA diet compared with the chow diet were significantly overrepresented in the PI3K/Akt pathway (10 genes, FDR = 0.001 ) and the HIF-1 signaling pathway (4 genes, FDR $=0.047$ ). The 67 genes downstream of TFs with significantly higher expression in the mNIA diet compared with the NIA diet were also significantly overrepresented in the PI3K/Akt pathway (9 genes, FDR $=0.002$ ). Increased HIF-1 expression is associated with upregulation of collagen isoforms and TIMPs in the heart, leading to increased fibrosis (51). The PI3K/ Akt pathway plays a key role in cardiac hypertrophy regulation of cardiac titin, stiffness, and angiogenesis (52). In combination with HIF-1 and VEGF, Akt contributes to pathological hypertrophy and angiogenesis (53). Furthermore, TGF- $\beta$ and TNF pathways, known to be profibrotic $(54,55)$, were significantly overrepresented in the mNIA diet compared with the chow and NIA. This suggests that higher dietary sucrose and fat result in changes in the transcriptional landscape of the murine heart to upregulate expression of genes in the HIF-1 and PI3K pathways that could lead to cardiac remodeling and increased cardiac fibrosis.

\section{Discussion}

The myocardium can change with age, often with increased cardiac fibrosis and hypertrophy, and these changes may eventually compromise cardiac function (1-4). Heart samples from the 2 longitudinal CR studies in NHPs provided an invaluable opportunity to examine the relationship between caloric intake and cardiac fibrosis. In fact, we determined that CR did not significantly alter cardiac fibrosis in NHPs in either the UW or NIA study. Instead, there was a dramatic approximately 5.9-fold increase in cardiac fibrosis in UW NHPs compared with NIA NHPs, consistent with the earlier report that both control and CR NHPs in the NIA study, with lower body weights and fasted blood glucose levels, were relatively cardioprotected compared with UW controls (21).

Gene expression analyses of ECM-associated targets, such as collagens, MMPs, and TIMPs, revealed significantly greater amounts of TIMP2 and TIMP4 in NIA CR hearts, compared with UW CR hearts. Furthermore, collagen type III expression was greater in UW CR, compared with NIA CR. Interestingly, MMP2 expression was also greater in NIA CR, compared with UW CR. High levels of plasma TIMP2 and TIMP4 have been documented in individuals with heart disease and heart failure (33). Although TIMPs are known to inhibit MMPs and slow down the breakdown of ECM proteins, TIMP2 activates certain MMPs, including proMMP2, in addition to inhibiting several MMP isoforms $(29,32)$. TIMP2 deletion models exhibit greater infarct size and increased LV dilation and dysfunction following MI (32). Therefore, it is unclear whether TIMP2 function is beneficial or harmful in the injured heart because it is now appreciated that MMPs and TIMPs regulate various inflammatory signaling pathways in addition to their classical roles in ECM turnover (29).

Earlier reports suggest that the differences in survival outcomes (i.e., why CR increased survival at UW and not NIA) of the 2 NHP studies were influenced by methodological differences $(21,24)$. Recently, emphasis has shifted to unraveling the precise elements of each study that help answer questions about underlying mechanisms. In the current study, we focus on diet composition, more specifically on the sucrose and fat content of each diet, to investigate cardiovascular contributions. Mitchell et al. pursued the hypothesis that diet composition and feeding regimen were primary drivers of the differing $\mathrm{CR}$ effect in the NHP studies and thus modeled the study design in mice. In that study, investigators analyzed measures of health, life span, metabolism, and gross pathology in ad libitum-fed, daily fasted, and CR groups of male mice. The factor with the greatest influence on health and life span was the duration of daily fasting, regardless of dietary composition (56).

Our findings establish a definitive link between diet composition and a prominent marker of cardiovascular health in primate LV samples from the 2 longitudinal NHP studies. Furthermore, we report cardiac 
fibrosis increased in mice following 6 months on a diet with higher sucrose and fat, a finding consistent with reports that a high-fat, high-sucrose Western diet increases cardiac fibrosis in other rodent models $(10,49)$. Although the UW diet had higher fat content than the NIA diet (Table 3), it was still well below the 35\% fat that is typical in a Western diet (25). Thus, we speculate that sucrose, a disaccharide composed of fructose and glucose, may be a central factor modulating age-associated cardiac fibrosis in the NHPs and mice in our study. In recent years, a multitude of investigations on the commensal microbiome and its influence on host health and disease have highlighted the crucial role the composition of the microbiome plays $(57,58)$. It has been shown that dietary composition shapes the microbiome and the relative abundance of various microbial species (58). In light of this, we reflect that the effect of the microbiome on cardiovascular health and cardiac fibrosis cannot be discounted. The microbiome would be a key factor for future studies investigating the influence of dietary composition on cardiac and metabolic health in our experimental models.

It is unknown how early the NHPs showed evidence of fibrosis, but the long-term diet exposure likely contributed to the greater fibrosis burden. Differences in degree of pathophysiology could be species specific or relative to length of time on the diet. Yet, the pattern of disease also differed because the cardiac fibrosis induced in the murine model after 6 months on the diet was predominantly perivascular, whereas LVs from both NHP studies showed interstitial and perivascular fibrosis. Interstitial fibrosis results in ventricular dilation and cardiomyocyte stiffness, playing a pathological role in heart failure $(59,60)$. The reasons for these differences are not clear but warrant further investigation in prospective longitudinal studies to assess cardiac function. A limitation of our study is that we did not perform comprehensive cardiac physiology assessments during the prolonged mouse feeding study. Future studies of these diets should include prospective serial noninvasive cardiac assessments as well as comprehensive metabolic analyses to establish a time course of the effect of dietary composition on cardiac pathology in the aging mammalian heart.

As a potentially first approach toward identifying the mechanisms contributing to understanding the pattern and extent of fibrosis in the mice, global transcriptomic analyses of the rodent tissues revealed distinct profiles associated with the types of diet: natural-ingredient/grain-based diets, semipurified/defined diets, or a natural-ingredient diet containing moderate fat and high sucrose. Interestingly, a grain-based diet with moderate fat and high sucrose appears to be associated with upregulation of metabolic pathways, including oxidative phosphorylation, as well as diet-induced cardiac stress. This indicates that diets higher in fat and sucrose may accelerate damage to an aging heart. Furthermore, the transcriptomic signature characterizing this grain-based, moderate-fat, high-sucrose diet (mNIA) was similarly different from both the grain-based and the standard diet, demonstrating that diet-induced cardiac stress has unique transcriptional and functional consequences, including the triggering of innate cardioprotective mechanisms, perhaps to counter the stress. However, to identify molecular mechanisms underlying diet-induced changes in cardiac pathology and to assess potential therapeutic targets, a deeper insight into the molecular and cellular changes in the heart mediated by dietary composition is warranted in future studies.

Cardiac fibrosis is a common pathological factor in many forms of heart failure (61). In HFpEF, the most common form of heart failure, it is a major underlying pathological finding $(62,63)$. HFpEF has risen dramatically in the past several decades, and this epidemic is, at least in part, driven by aging and the increase in metabolic syndrome $(62,64)$. Because increased sucrose consumption over the past several decades is strongly associated with the recent metabolic syndrome epidemic (65-67), this raises the intriguing hypothesis that dietary sucrose may drive fibrosis in the aging heart and contribute to the increase in HFpEF.

\section{Methods}

The data and pipelines from our RNA-Seq experiments have been deposited into the National Center for Biotechnology Information's Gene Expression Omnibus, accession number GSE135532. All the analyses were performed in a randomized and blinded manner.

Subjects. Male 6-week-old C57BL/6 mice were obtained from Charles Rivers Laboratory and housed at the Harvard BRI under a 12-hour light/12-hour dark cycle and given unrestricted access to food and water. The animals were randomly assigned to one of 4 diets: standard rodent chow (Prolab IsoPro RMH 3000 5P75, LabDiet), NIA primate diet (5L1F, LabDiet), UW primate diet (Envigo TD.85387, Harlan Laboratories), or mNIA diet (NIA diet modified to have sucrose and fat content matching the UW diet; Table 3 and refs. 17, 21, 23) for 6 months.

To measure fasting glucose, animals were fasted for at least 5 hours, and blood glucose was measured using a handheld glucometer (Contour 9545C, Ascensia Diabetes Care) with blood from the tail vein. Five-hour-fasted 
plasma was used to measure insulin levels using mouse ultrasensitive insulin ELISA kit from ALPCO according to the manufacturer's instructions. Animals were weighed and then euthanized by isoflurane overdose followed by exsanguination. Hearts were collected, weighed, and bisected. One-half was used for histological analyses (paraformaldehyde preserved) and the other half was used for RNA-Seq (liquid nitrogen frozen).

Hepatic and plasma triglycerides. Triglyceride content of liver and 5-hour fasting plasma was measured using a colorimetric triglyceride quantification kit from BioVision. A piece of liver was weighed and homogenized in $5 \% \mathrm{NP}-40$ in water and heated to $80^{\circ} \mathrm{C}$ for 5 minutes to solubilize all the triglyceride. Liver samples were diluted 20 -fold in water, and triglyceride content was estimated using the colorimetric assay according to the manufacturer's instructions.

Histology. NHP LV samples were previously harvested from the ongoing longitudinal studies at the NIA and UW. Samples were flash frozen at collection and stored at $-80^{\circ} \mathrm{C}$ until processing. Approximately 5 -mm pieces were cut from the tissue and frozen in OCT in tissue molds and then sectioned on a cryostat (Leica CM 1950) to obtain 10- $\mu \mathrm{m}$ sections. Mouse hearts for histology were fixed in $4 \%$ paraformaldehyde for a period of 24 hours and then transferred to $70 \%$ ethanol, followed by dehydration and embedding in paraffin and sectioning (5- $\mu \mathrm{m}$ sections).

Masson's trichrome staining. Masson's trichrome staining to measure fibrosis in NHP LV sections (frozen sections, $10 \mu \mathrm{m}$ thick) was performed as follows. Sections were fixed overnight at room temperature in Bouin's fixative. After rinsing in tap water to remove residual Bouin's fixative, sections were stained for 10 minutes with a 1:1 mixture of hematoxylin A and B (A, catalog 88028, and B, catalog 88029; Thermo Scientific). Scarlet acid stain (catalog 26367-04, Electron Microscopy Sciences) was added for 10 minutes, followed by 5 minutes in phosphomolybdic acid solution (catalog 26367-05, Electron Microscopy Sciences). The sections were then incubated in aniline blue solution (catalog 26367-06, Electron Microscopy Sciences) for 5 minutes and then transferred to $1 \%$ acetic acid for 5 minutes. This was followed by dehydration in ethanol and xylene; the sections were then mounted in permount (catalog 17986-01, Electron Microscopy Sciences) and cured for 24 hours before imaging. Stained sections were imaged using Zeiss AxioScan imaging system at the Harvard Center for Biological Imaging. Total fibrosis was calculated using ImageJ (NIH) as previously described (27). Masson's trichrome staining of mouse heart sections (paraffin sections, $5 \mu \mathrm{m}$ thick) was performed following a similar protocol, with 6 minutes in aniline blue.

RNA isolation and expression profiling from NHP tissue. RNA was isolated from NHP hearts using Ribozol (phenol/chloroform extraction, ethanol precipitation; catalog 97064-952, VWR Life Science) and subjected to quality control on a bioanalyzer (Agilent Bioanalyzer 2100). nCounter gene expression profiling was used to assess the expression of ECM proteins, MMPs, and TIMPs. The nCounter gene expression assay is a robust method for the detection of gene expression by direct imaging using color-coded probe pairs specific to target genes $(30,68)$. Target mRNAs of interest are detected using a pair of reporter and capture probes that are specific for an approximately 100-bp region of the target. Reporter probes have a color code, while capture probes have a biotin label to facilitate downstream attachment and detection of targets ( 30 , 68). One hundred nanograms of RNA adjusted for degradation using DV200 values obtained from the tape station was used as input for gene expression profiling using nCounter gene expression assays. Hybridization of RNA and probes was performed at $67^{\circ} \mathrm{C}$ for 18 hours, followed by purification and binding to the imaging membrane using the NanoString prep station. Finally, imaging and analyses of counts were performed using nSolver 4.0 software platform as previously described (30).

Transcript abundances were standardized using calibration controls and normalized using positive-control housekeeping genes as previously described (30). We quantified expression levels of 19 genes for 4 cohorts: NIA control, NIA CR, UW control, and UW CR. For each of the 4 cohorts, 6 LV samples were assayed. In the heatmap, we visualize the median (across the $6 \mathrm{LV}$ samples) normalized abundances for the transcripts across the cohorts. Because the expression levels are gene specific, and our goal was to compare transcript abundances across the 4 cohorts, we further normalized per transcript by centering and scaling across the 4 cohorts.

$R N A$ isolation and sequencing. Total RNA from the lower half of the mouse heart ventricles was isolated using Ribozol. Four biological replicates were used per diet; each replicate represents myocardium from a single animal. Quality control of RNA samples was performed by tape station; samples with RIN greater than 7.0 were processed for library preparation and qPCR. Single-end (75 bp) sequencing was performed using an Illumina NextSeq $1 \times 75$ high-output flow cell. Quality control, poly-adenylated mRNA isolation, library preparation, qPCR, and sequencing were performed by the Harvard FAS center for systems biology. 
Analyses of RNA-Seq data. Total poly-adenylated mRNA was isolated and sequenced on an Illumina NextSeq flow cell in high-output mode to generate approximately 305 million $1 \times 75$ bp reads. We then used a standard 3-part pipeline comprising (a) alignment of reads to the mouse transcriptome (RefSeq transcript database: mm10), (b) quantification of gene expression data from the aligned reads using an expectation-maximization algorithm, and (c) differential expression analyses using DESeq2 (39). Our pipeline is summarized in Supplemental Figure 3A.

Briefly, short reads were aligned to the mouse transcriptome using the Burrows-Wheeler aligner MEM algorithm with default parameters (36). We then quantified gene expression levels (gene-level counts) using a well-established expectation-maximization algorithm (37). Differential expression analyses were performed using DESeq2 (39). A parametric fit with default parameters was used, $P$ values were calculated using a Wald's test, and these $P$ values were adjusted for multiple comparisons using the Benjamini-Hochberg procedure to obtain FDRs. All diets were compared in a pairwise fashion to obtain differentially expressed transcripts (4 diets: chow, UWM, NIA, and mNIA; 6 pairwise comparisons). To validate that our analyses were robust to the choice of alignment algorithm, we also used a different aligner - STAR (69) — and found that our results remained unchanged (Supplemental Figure $3 C)$. Further, our results also remained unaltered if gene-level counts instead of transcript-levels counts were used (Supplemental Figure 3D).

For any diet pair, we defined genes as being significantly differentially expressed if they had (a) a fold change (FC) of at least 1.5 and (b) an FDR (after Benjamini-Hochberg multiple-testing correction) less than 0.1. In Figure 3C, all transcripts in the volcano plot presented in color are based on an FC > 1.5 and $P<0.05$ threshold (rather than an FDR $<0.1$ threshold). This provides insights into diet pairs that had the greatest transcriptomic changes (at slightly more permissive thresholds). However, for all analyses including overrepresentation and network analyses, we used the more stringent FC $>1.5$ and FDR $<0.1$ thresholds. Figure 3D presents the numbers of significantly differentially expressed transcripts at these stringent thresholds.

Overrepresentation and network analyses. For overrepresentation analyses, we used only genes that were significantly differentially expressed, i.e., FC $\geq 1.5$ and FDR $<0.1$. This represents a stringent cutoff because (a) we used both an effect size and a significance threshold, and (b) the significance threshold was based on FDR rather than on $P$ values. To evaluate whether these significantly differentially expressed genes were overrepresented in KEGG pathways, we used a hypergeometric test via the WebGestalt interface (40). We considered a pathway significantly overrepresented if the FDR (i.e., $P$ value computed using a hypergeometric test followed by Benjamini-Hochberg multiple-testing correction) associated with that pathway was less than 0.15. This is again an independent stringent cutoff because the significance threshold was based on the FDR rather than on $P$ values. However, 10/14 reported pathways had at least 5-fold enrichment and an FDR less than 0.05 (Supplemental Table 2). Further, even if we went to an FDR threshold of less than 0.005 , the majority of pathways (8/14 pathways) still passed this threshold (Supplemental Table 2). Thus, our results hold at much more stringent FC and FDR thresholds.

Further, most of the FDRs obtained were highly significant $(P<0.001$, Supplemental Table 2). We used stringent thresholds both for the selection of differentially expressed genes and to evaluate significant overrepresentation to minimize the false positive rate and have a very high degree of confidence regarding the reported pathways (i.e., pathways overrepresented by significantly differentially expressed genes). However, we may be underpowered to detect all pathways significantly overrepresented for differentially expressed genes. This framework fits the paradigm of our study, where we are interested in focusing on the most robust hits but make no claims regarding being able to discover all pathways of interest.

For the protein network analyses, we used high-quality Mus musculus protein-protein interactions from the HINT database (42). The interactions were systematically curated from the literature (both small-scale and high-throughput experiments) using well-established criteria and are highly reliable because each interaction is supported by at least 2 independent experimental assays (42).

Statistics. Statistical analyses were performed on MATLAB. Kruskal-Wallis tests were used to compare data across experimental groups. Additionally, for pairwise comparisons, 2-sided Mann-Whitney $U$ tests were used with appropriate Bonferroni's corrections to adjust for multiple comparisons. Significance level was set at $P<0.05$.

Study approval. All animal studies were reviewed and approved by the Harvard University Institutional Animal Care and Use Committee, Cambridge, Massachusetts, USA (protocol 16-05-273). 
All experiments were conducted in accordance with the Guide for the Care and Use of Laboratory Animals (National Academies Press, 2011).

\section{Author contributions}

RTL, JAM, and RJC conceived the study. JAM, RJC, and RTL participated in study design and supervision of the project. NN, AV, ACW, KKP, SHK, EMRB, and AYZ carried out experiments in the Lee Laboratory. KLV collected data and samples at the NIA and edited the manuscript. NN, AV, JD, ACW, KKP, and SHK analyzed the data. RNA-Seq data analysis and statistical analyses were performed by JD. All authors reviewed and approved the final manuscript, which was initially drafted by NN, AV, JD, and RTL.

\section{Acknowledgments}

This work was supported by grants AG047131, AG059129, HL119230 from NIH and a Leducq Foundation TransAtlantic Network grant (to RTL). The NIA study was supported by the Intramural Research Program of the NIA, NIH. The UW study was supported by R01AG040178 (to RJC) and was made possible in part by National Center for Research Resources/Office of Research Infrastructure Programs grants P51RR000167/P51OD011106 to the Wisconsin National Primate Research Center, UW. We thank the Harvard Center for Biological Imaging for infrastructure and support.

Address correspondence to: Richard T. Lee, Department of Stem Cell and Regenerative Biology, Harvard University, Sherman Fairchild Biochemistry Building 159, 7 Divinity Ave., Cambridge, Massachusetts 02138, USA. Phone: 617.496.5394; Email: richard_lee@harvard.edu.

1. North BJ, Sinclair DA. The intersection between aging and cardiovascular disease. Circ Res. 2012;110(8):1097-1108

2. Fleg JL, Strait J. Age-associated changes in cardiovascular structure and function: a fertile milieu for future disease. Heart Fail Rev. 2012;17(4-5):545-554.

3. Strait JB, Lakatta EG. Aging-associated cardiovascular changes and their relationship to heart failure. Heart Fail Clin. 2012;8(1):143-164.

4. Steenman M, Lande G. Cardiac aging and heart disease in humans. Biophys Rev. 2017;9(2):131-137.

5. Benjamin EJ, et al. Heart disease and stroke statistics - 2018 update: a report from the American Heart Association. Circulation. 2018;137(12):e67-e492.

6. Biernacka A, Frangogiannis NG. Aging and cardiac fibrosis. Aging Dis. 2011;2(2):158-173.

7. Travers JG, Kamal FA, Robbins J, Yutzey KE, Blaxall BC. Cardiac fibrosis: the fibroblast awakens. Circ Res. 2016;118(6):1021-1040.

8. Mohammed SF, Hussain S, Mirzoyev SA, Edwards WD, Maleszewski JJ, Redfield MM. Coronary microvascular rarefaction and myocardial fibrosis in heart failure with preserved ejection fraction. Circulation. 2015;131(6):550-559.

9. Cavalera M, Wang J, Frangogiannis NG. Obesity, metabolic dysfunction, and cardiac fibrosis: pathophysiological pathways, molecular mechanisms, and therapeutic opportunities. Transl Res. 2014;164(4):323-335.

10. Aguila MB, Mandarim-de-Lacerda CA. Blood pressure, ventricular volume and number of cardiomyocyte nuclei in rats fed for 12 months on diets differing in fat composition. Mech Ageing Dev. 2001;122(1):77-88.

11. Fontana L. Modulating human aging and age-associated diseases. Biochim Biophys Acta. 2009;1790(10):1133-1138.

12. Heidemann C, Schulze MB, Franco OH, van Dam RM, Mantzoros CS, Hu FB. Dietary patterns and risk of mortality from cardiovascular disease, cancer, and all causes in a prospective cohort of women. Circulation. 2008;118(3):230-237.

13. Speakman JR, Mitchell SE. Caloric restriction. Mol Aspects Med. 2011;32(3):159-221.

14. AlGhatrif M, et al. Beneficial cardiac effects of caloric restriction are lost with age in a murine model of obesity. J Cardiovasc Transl Res. 2013;6(3):436-445.

15. Anderson RM, Weindruch R. Metabolic reprogramming, caloric restriction and aging. Trends Endocrinol Metab. 2010;21(3):134-141.

16. Weiss EP, Fontana L. Caloric restriction: powerful protection for the aging heart and vasculature. Am J Physiol Heart Circ Physiol. 2011;301(4):H1205-H1219.

17. Colman RJ, Beasley TM, Kemnitz JW, Johnson SC, Weindruch R, Anderson RM. Caloric restriction reduces age-related and all-cause mortality in rhesus monkeys. Nat Commun. 2014;5:3557.

18. Fontana L, Partridge L, Longo VD. Extending healthy life span — from yeast to humans. Science. 2010;328(5976):321-326.

19. Fontana L, Meyer TE, Klein S, Holloszy JO. Long-term calorie restriction is highly effective in reducing the risk for atherosclerosis in humans. Proc Natl Acad Sci U S A. 2004;101(17):6659-6663.

20. Sohal RS, Weindruch R. Oxidative stress, caloric restriction, and aging. Science. 1996;273(5271):59-63.

21. Mattison JA, et al. Caloric restriction improves health and survival of rhesus monkeys. Nat Commun. 2017;8:14063.

22. Colman RJ, et al. Caloric restriction delays disease onset and mortality in rhesus monkeys. Science. 2009;325(5937):201-204

23. Mattison JA, et al. Impact of caloric restriction on health and survival in rhesus monkeys from the NIA study. Nature. 2012;489(7415):318-321.

24. Vaughan KL, Kaiser T, Peaden R, Anson RM, de Cabo R, Mattison JA. Caloric restriction study design limitations in rodent and nonhuman primate studies. J Gerontol A Biol Sci Med Sci. 2017;73(1):48-53.

25. Wang Z, Li L, Zhao H, Peng S, Zuo Z. Chronic high fat diet induces cardiac hypertrophy and fibrosis in mice. Metab Clin Exp. 2015;64(8):917-925 
26. Collins TJ. ImageJ for microscopy. BioTechniques. 2007;43(supp1 1):25-30.

27. Teekakirikul $\mathrm{P}$, et al. Cardiac fibrosis in mice with hypertrophic cardiomyopathy is mediated by non-myocyte proliferation and requires Tgf- $\beta$. J Clin Invest. 2010;120(10):3520-3529.

28. Löffek S, Schilling O, Franzke CW. Series "matrix metalloproteinases in lung health and disease": biological role of matrix metalloproteinases: a critical balance. Eur Respir J. 2011;38(1):191-208.

29. Arpino V, Brock M, Gill SE. The role of TIMPs in regulation of extracellular matrix proteolysis. Matrix Biol. 2015;44-46:247-254.

30. Geiss GK, et al. Direct multiplexed measurement of gene expression with color-coded probe pairs. Nat Biotechnol. 2008;26(3):317-325.

31. Koskivirta I, et al. Mice with tissue inhibitor of metalloproteinases 4 (Timp4) deletion succumb to induced myocardial infarction but not to cardiac pressure overload. J Biol Chem. 2010;285(32):24487-24493.

32. Kandalam V, et al. TIMP2 deficiency accelerates adverse post-myocardial infarction remodeling because of enhanced MT1MMP activity despite lack of MMP2 activation. Circ Res. 2010;106(4):796-808.

33. Zile MR, Baicu CF. Biomarkers of diastolic dysfunction and myocardial fibrosis: application to heart failure with a preserved ejection fraction. J Cardiovasc Transl Res. 2013;6(4):501-515.

34. Leite $\mathrm{RD}$, et al. Resistance training may concomitantly benefit body composition, blood pressure and muscle MMP-2 activity on the left ventricle of high-fat fed diet rats. Metab Clin Exp. 2013;62(10):1477-1484.

35. Dewey FE, Rosenthal D, Murphy DJ, Froelicher VF, Ashley EA. Does size matter? Clinical applications of scaling cardiac size and function for body size. Circulation. 2008;117(17):2279-2287.

36. Li H, Durbin R. Fast and accurate short read alignment with Burrows-Wheeler transform. Bioinformatics. 2009;25(14):1754-1760.

37. Xing Y, Yu T, Wu YN, Roy M, Kim J, Lee C. An expectation-maximization algorithm for probabilistic reconstructions of fulllength isoforms from splice graphs. Nucleic Acids Res. 2006;34(10):3150-3160.

38. van der Maaten L, Hinton G. Visualizing data using t-SNE. J Mach Learning Res. 2008;9:2579-2605.

39. Love MI, Huber W, Anders S. Moderated estimation of fold change and dispersion for RNA-seq data with DESeq2. Genome Biol. 2014;15(12):550.

40. Wang J, Duncan D, Shi Z, Zhang B. WEB-based GEne SeT AnaLysis Toolkit (WebGestalt): update 2013. Nucleic Acids Res. 2013;41(Web Server issue):W77-W83.

41. Kanehisa M, Goto S. KEGG: Kyoto Encyclopedia of Genes and Genomes. Nucleic Acids Res. 2000;28(1):27-30.

42. Das J, Yu H. HINT: high-quality protein interactomes and their applications in understanding human disease. BMC Syst Biol. 2012;6:92.

43. Das J, Gayvert KM, Bunea F, Wegkamp MH, Yu H. ENCAPP: elastic-net-based prognosis prediction and biomarker discovery for human cancers. BMC Genomics. 2015;16:263.

44. Das J, et al. Cross-species protein interactome mapping reveals species-specific wiring of stress response pathways. Sci Signal 2013;6(276):ra38.

45. Vo TV, et al. A proteome-wide fission yeast interactome reveals network evolution principles from yeasts to human. Cell. 2016;164(1-2):310-323.

46. Wang X, Wei X, Thijssen B, Das J, Lipkin SM, Yu H. Three-dimensional reconstruction of protein networks provides insight into human genetic disease. Nat Biotechnol. 2012;30(2):159-164.

47. Han H, et al. TRRUST v2: an expanded reference database of human and mouse transcriptional regulatory interactions. Nucleic Acids Res. 2018;46(D1):D380-D386.

48. Jandeleit-Dahm K, Cooper ME. The role of AGEs in cardiovascular disease. Curr Pharm Des. 2008;14(10):979-986.

49. Tikellis C, et al. Cardiac inflammation associated with a Western diet is mediated via activation of RAGE by AGEs. Am J Physiol Endocrinol Metab. 2008;295(2):E323-E330.

50. Li N, Bian H, Zhang J, Li X, Ji X, Zhang Y. The Th17/Treg imbalance exists in patients with heart failure with normal ejection fraction and heart failure with reduced ejection fraction. Clin Chim Acta. 2010;411(23-24):1963-1968.

51. Warbrick I, Rabkin SW. Hypoxia-inducible factor 1-alpha (HIF-1 $\alpha$ ) as a factor mediating the relationship between obesity and heart failure with preserved ejection fraction. Obes Rev. 2019;20(5):701-712.

52. Gladden JD, Linke WA, Redfield MM. Heart failure with preserved ejection fraction. Pflugers Arch. 2014;466(6):1037-1053.

53. Aoyagi T, Matsui T. Phosphoinositide-3 kinase signaling in cardiac hypertrophy and heart failure. Curr Pharm Des. 2011;17(18):1818-1824.

54. Khalil H, et al. Fibroblast-specific TGF- $\beta-S m a d 2 / 3$ signaling underlies cardiac fibrosis. J Clin Invest. 2017;127(10):3770-3783.

55. Zhang W, et al. The development of myocardial fibrosis in transgenic mice with targeted overexpression of tumor necrosis factor requires mast cell-fibroblast interactions. Circulation. 2011;124(19):2106-2116.

56. Mitchell SJ, et al. Daily fasting improves health and survival in male mice independent of diet composition and calories. Cell Metab. 2019;29(1):221-228.e3.

57. Voreades N, Kozil A, Weir TL. Diet and the development of the human intestinal microbiome. Front Microbiol. $2014 ; 5: 494$.

58. Flint HJ, Scott KP, Louis P, Duncan SH. The role of the gut microbiota in nutrition and health. Nat Rev Gastroenterol Hepatol. 2012;9(10):577-589.

59. Dai Z, Aoki T, Fukumoto Y, Shimokawa H. Coronary perivascular fibrosis is associated with impairment of coronary blood flow in patients with non-ischemic heart failure. J Cardiol. 2012;60(5):416-421.

60. van Heerebeek L, Paulus WJ. Understanding heart failure with preserved ejection fraction: where are we today? Neth Heart $J$. 2016;24(4):227-236.

61. Segura AM, Frazier OH, Buja LM. Fibrosis and heart failure. Heart Fail Rev. 2014;19(2):173-185.

62. Bhuiyan T, Maurer MS. Heart failure with preserved ejection fraction: persistent diagnosis, therapeutic enigma. Curr Cardiovasc Risk Rep. 2011;5(5):440-449.

63. Heart Failure Society of America, et al. HFSA 2010 comprehensive heart failure practice guideline. J Card Fail. 2010;16(6):e1-194.

64. Carbone S, Popovic D, Lavie CJ, Arena R. Obesity, body composition and cardiorespiratory fitness in heart failure with preserved ejection fraction [published online ahead of print August 10, 2017]. Future Cardiol. https://doi.org/10.2217/ fca-2017-0023. 
65. Malik VS, Popkin BM, Bray GA, Despres JP, Willett WC, Hu FB. Sugar-sweetened beverages and risk of metabolic syndrome and type 2 diabetes: a meta-analysis. Diabetes Care. 2010;33(11):2477-2483.

66. DiNicolantonio JJ, O'Keefe JH, Lucan SC. Added fructose: a principal driver of type 2 diabetes mellitus and its consequences. Mayo Clin Proc. 2015;90(3):372-381.

67. Yang Q, Zhang Z, Gregg EW, Flanders WD, Merritt R, Hu FB. Added sugar intake and cardiovascular diseases mortality among US adults. JAMA Intern Med. 2014;174(4):516-524.

68. Kulkarni MM. Digital multiplexed gene expression analysis using the NanoString nCounter system. Curr Protoc Mol Biol. 2011;94(1):25B.10.1-25B.10.17.

69. Dobin A, et al. STAR: ultrafast universal RNA-seq aligner. Bioinformatics. 2013;29(1):15-21. 